$\begin{array}{cl}\text { PRAMANA } & \text { (c) Indian Academy of Sciences } \\ \begin{array}{c}\text { journal of } \\ \text { physics }\end{array} & \begin{array}{l}\text { Vol. 53, No. } 6 \\ \text { December } 1999 \\ \text { pp. 1-12 }\end{array}\end{array}$

\title{
Gravitational collapse and naked singularities
}

\author{
Tomohiro Harada \\ Astronomy Unit, School of Mathematical Sciences, Queen Mary, University of London, Mile End \\ Road, London E1 4NS, UK
}

\begin{abstract}
Gravitational collapse is one of the most striking phenomena in gravitational physics. The cosmic censorship conjecture has provided strong motivation for research in this field. In the absence of general proof for censorship, many examples have been proposed, in which naked singularity is the outcome of gravitational collapse. Recent development has revealed that there are examples of naked singularity formation in the collapse of physically reasonable matter fields, although the stability of these examples is still uncertain. We propose the concept of 'effective naked singularities', which will be quite helpful because general relativity has the limitation of its application for high-energy end. The appearance of naked singularities is not detestable but can open a window for the new physics of strongly curved spacetimes.
\end{abstract}

Keywords. general relativity, spacetime singularity

PACS Nos 04.20.Dw

\section{Introduction}

If a pressure gradient force is not sufficiently strong, a body can continue collapsing due to its self-gravity. This phenomenon is called gravitational collapse. The astrophysical relevance of gravitational collapse is now robust. We have observational evidence for massive and/or supermassive black holes. It is well understood that there exists an upper limit to the maximum possible mass of a spherical body of cold nuclear matter. We can also naturally consider that black holes may have formed from cosmological perturbations in the early stages of universe.

The singularity theorems (see, e.g., [1]) state that there exist spacetime singularities in generic gravitational collapse. At singularities the smoothness of the spacetime metric is lost. More precisely, a singularity is not regarded as a point in the spacetime manifold but one in the boundary of spacetime (see [2]). Classical physics cannot be applied to spacetime singularities because classical physics implicitly assumes the smoothness of spacetime, where and hereafter I refer to all physics which do not contain quantum gravity as classical physics. This implies the limitation of classical physics.

In spite of this limitation, it is possible to have some kind of future predictability using only classical physics if all singularities are hidden from physically relevant regions. Penrose [3] conjectured that a system which evolves, according to classical general relativity with reasonable equations of state, from generic non-singular initial data on a suitable Cauchy hypersurface, does not develop any spacetime singularity which is visible from 
infinity. This conjecture is actually a weak version of censorship. If this conjecture is true, we can predict the whole evolution using classical physics for the spacetime region outside black holes. The strong version [4] states that a physically reasonable classical spacetime is globally hyperbolic. This conjecture claims that classical physics can predict the evolution of the whole spacetime. A singularity which is censored by the strong version of censorship is called a naked singularity. A naked singularity which is censored by the weak version is called a globally naked singularity. A naked singularity which is not a globally naked singularity is called a locally naked singularity.

The cosmic censorship has not been proved yet in spite of a huge amount of effort. It is clear that there is ambiguity in the definition of physical reasonableness. We can consider two aspects of physical reasonableness, one for matter fields and the other for initial data. For matter fields, many people have considered that energy conditions should be satisfied. Furthermore, we can consider more concrete models, such as a perfect fluid with reasonable equations of state, elementary fields, and so on. For initial data, we should restrict our attention to non-singular initial data. Moreover, we can consider sufficiently smooth initial data. The initial data should be generic in some appropriate topology.

It is true that the cosmic censorship conjecture has long provided researchers with strong motivation into studies of gravitational collapse but I think now that we should regard it as one of aspects in gravitational collapse physics. I would like to come back to the original standpoint. How is physical gravitational collapse? How do black holes form? How do spacetime singularities form?

\section{Spherically symmetric dust collapse}

The spherically symmetric collapse of a dust fluid is the simplest nontrivial example of gravitational collapse. Dust means a fluid with vanishing pressure. This model is pedagogical to understand how the situation gets complicated if we include inhomogeneity in the model.

\subsection{Homogeneous dust ball}

In the spherical collapse of a homogeneous dust ball, the dust interior and vacuum exterior are described by the collapsing Friedmann solution and the Schwarzschild solution, respectively. This collapse solution, which is called the Oppenheimer-Snyder solution [5], has given a picture of a black hole as a final state of gravitational collapse. The mass $M$ of the dust ball begins to collapse and the radius of the dust ball gets smaller and smaller. At some moment it equals the Schwarzschild radius $2 M$, which corresponds to the formation of an event horizon. During this process, the redshift of the light ray which is emitted at the surface increases and becomes infinite at the formation of an event horizon. Subsequently, a singularity forms everywhere at the same time in the dust interior. The singularity is spacelike and completely hidden by the horizon. No causal curve can emanate from the singularity. The causal structure of this spacetime is depicted in Fig. 1. This picture is generally accepted as a final fate of gravitational collapse in realistic situations. 


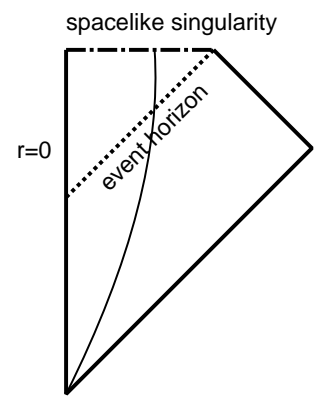

Figure 1. Spacetime diagram of the collapse of a homogeneous model.

\subsection{Inhomogeneous dust ball}

The situation is completely different if we allow inhomogeneity in the model. The collapse of an inhomogeneous dust ball is described by an exact solution, the Lemaitre-TolmanBondi (LTB) solution [6]. This solution is general in a sense that it has two arbitrary functions, which correspond to initial density and velocity profiles. In this solution, a singularity forms at the centre, which is called a shell-focusing singularity. The shellfocusing singularity can be a naked singularity. This was first found numerically [7] and subsequently proved mathematically [8]. The conditions for appearance of naked singularities have been extensively investigated [9]. The result is that naked singularities are the generic outcome of this collapse models which develop both from non-singular initial data and from sufficiently smooth initial data. The naked singularity is globally naked singularities or locally naked singularities, depending on the choice of the arbitrary functions. The causal structure is depicted in Fig. 2.

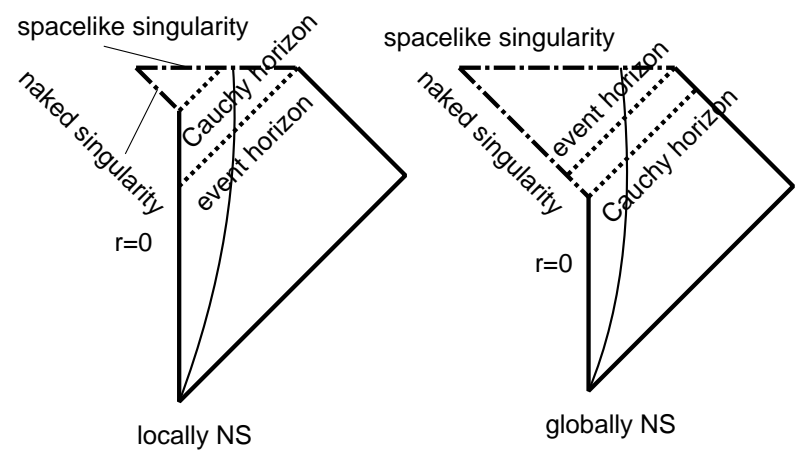

Figure 2. Spacetime diagram of the collapse of a inhomogeneous model.

For a while we restrict our attention to marginally bound collapse with initial data where the initial density profile is given by an analytic function with respect to the locally Cartesian coordinates. In this case, the structure of naked shell-focusing singularity has been revealed. The redshift of the first null ray which emanates from the singularity is finite but becomes infinite immediately for later null rays [8]. This naked singularity is curvature strong $[10,11]$. From this singularity, not only radial null rays but also nonradial null rays 
emanate, which implies that the singularity is not point-like [12,13].

From this structure of the naked shell-focusing singularities, it is undoubted that they are genuine and inextendible singularities. On the other hand, the dust fluid would not be physically reasonable because vanishing pressure should not be physically accepted. The recent development in gravitational collapse physics has provided modern examples of naked singularities where matter fields are sufficiently physically reasonable.

\section{Modern examples of naked singularities}

The problem of gravitational collapse involves dynamical and inhomogeneous spacetimes in its nature. It is difficult to obtain somewhat general exact solutions in this situation except for in limited systems such as the LTB solutions. Hence, it is natural to try to understand the qualitative nature of gravitational collapse by numerical simulations. This approach is getting more and more popular in the progress of numerical relativity and has a potential to discover new unexpected phenomena in general relativity. One of the most successful ones will be critical behaviour at the black hole threshold.

\subsection{Black hole threshold}

The critical behaviour in gravitational collapse was discovered in a spherical system of a massless scalar field [14], subsequently has been found in a variety of systems, such as axisymmetric gravitational waves [15], spherical system of a radiation fluid [16], spherical system of a perfect fluid with the equation of state $P=k \rho$ [17], and so on. Suppose we have a one-parameter family of initial data sets parametrised by a parameter $p$, which evolve through the Einstein equations, and the system has two extremal end states, one is a collapse to a black hole and the other is a dispersion to infinity. For instance, the data with a sufficiently large value for $p$ evolves to a black holes and the one with a sufficiently small value for $p$ evolves to a dispersion. Then, there should be a critical value $p^{*}$ at the threshold between two end states. For a near-critical case $p \approx p^{*}$, the collapse first approaches a self-similar solution, which is called a critical solution, and then it deviates away from the critical solution. For a supercritical and near-critical case, a black hole forms finally and the mass $M_{B H}$ of the formed black hole satisfies the scaling law $M_{B H} \propto\left|p-p^{*}\right|^{\gamma}$, where $\gamma$ is a positive constant called a critical exponent. These phenomena are universal to the choice of the one-parameter family of initial data sets.

This phenomenon is well described by the intermediate behaviour around the intermediate attractor [18]. The critical solution is identified with a self-similar solution with a single unstable mode. Here we consider a space of functions of $x$, where we put $x=\ln [r /(-t)]$ and $\tau=-\ln (-t)$ for some appropriate time and radial coordinates, $t$ and $r$. Then a selfsimilar solution corresponds to a fixed point, while a non self-similar solution moves along a curve in this space as $\tau$ increases. The critical solution is a fixed point with a single unstable direction. In other words, this fixed point has a stable manifold of codimension one, which is called a critical surface. A one-parameter family of initial data sets, which correspond to a curve in this space, generically has an intersection with the critical surface. Since the intersection evolves to the critical solution, the intersection corresponds to the 
data with the critical value $p^{*}$. For a near critical case, $p \approx p^{*}$, the initial data near the intersection first shadows the critical collapse but deviates away later because of the growth of the unstable mode. It can be shown that the critical exponent $\gamma$ is equal to the inverse of the eigenvalue of the unstable mode. The value calculated from the eigenvalue analysis shows a good agreement with the value obtained from the results of numerical simulations.

For the supercritical collapse, the formed black hole mass $M_{B H}$ is proportional to $\mid p-$ $\left.p^{*}\right|^{\gamma}$. From a dimensional consideration, we can derive that the curvature strength scales as $M_{B H}{ }^{-2}$ in the region just outside the event horizon. Then, let us consider the limit to the critical collapse from the supercritical collapse, i.e., $p \rightarrow p^{*}$. In this limit, the black hole mass approaches zero and the curvature just outside the event horizon is going to infinity. Since we have arbitrarily strong curvature outside the event horizon by fine-tuning, the black hole threshold can be regarded as a naked singularity. This intuitive consideration is confirmed by more straightforward work [19], in which the causal structure of the critical solution found in the spherical system of a massless scalar field was shown to be nakedsingular and given by one of the two possible spacetime diagrams depicted in Fig. 3.
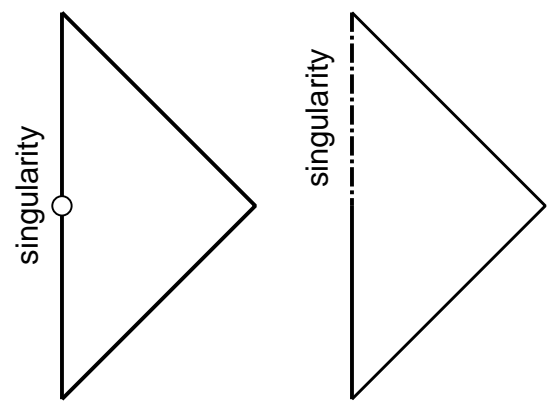

Figure 3. Spacetime diagram of the critical solution found in the spherical collapse of a massless scalar field.

It is now clear that critical collapse at the black hole threshold provides examples of naked singularities which form in the collapse of physically reasonable matter fields with non-singular initial data. However, it is also clear that the critical collapse is unstable and realised only as a result of exact fine-tuning. This implies that the formation of these naked singularities is not generic.

\subsection{Self-similar attractor}

As we have seen, critical behaviour in gravitational collapse is associated with a selfsimilar solution with a single unstable mode. It is expected that if we have a self-similar solution with no unstable mode, it will act as an attractor.

In fact, this is the case for a perfect fluid with the equation of state $P=k \rho$ for $0<k \leq 0.03$. In the result of numerical simulations [20], it was observed that a spherically collapsing perfect fluid approaches a self-similar solution from many initial data sets without any fine-tuning of initial data parameters. The approached solution is different from the critical solution and turns out to be one of the discrete set of self-similar solutions, which was previously discovered numerically [21]. This solution is called the general relativistic Larson-Penston solution. This solution is stable against spherical linear perturbations, 
while other self-similar solutions are unstable against those perturbations [20,22]. These results strongly suggest that the general relativistic Larson-Penston self-similar solution acts as an attractor in the gravitational collapse of a perfect fluid.

The general relativistic Larson-Penston solution is interesting in the context of cosmic censorship [21]. This solution describes the formation of naked singularity in the collapse of a perfect fluid from sufficiently smooth initial condition for $0<k \lesssim 0.0105$. This singularity can be globally naked after an appropriate matching to the Schwarzschild solution as is depicted in Fig. 4. For $k \gtrsim 0.0105$, this singularity is spacelike.

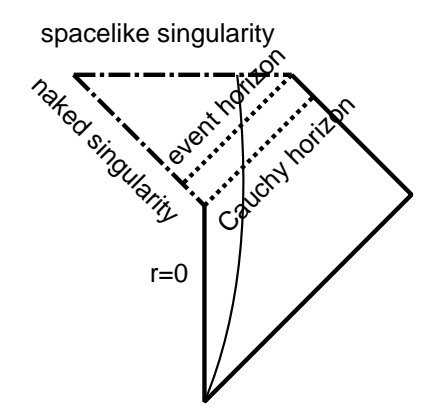

Figure 4. Spacetime diagram of the general relativistic Larson-Penston solution.

Since the general relativistic Larson-Penston solution is an attractor solution, the naked singularity formation is the outcome of generic gravitational collapse of a perfect fluid with the equation of state $P=k \rho(0<k \lesssim 0.0105)$ in spherical symmetry. No fine-tuning is needed to realise the naked singularity unlike in the black hole threshold. This is also consistent with the result of numerical simulations [23]. As a result, if we can restrict our attention to spherically symmetric collapse, we can conclude generic violation of cosmic censorship.

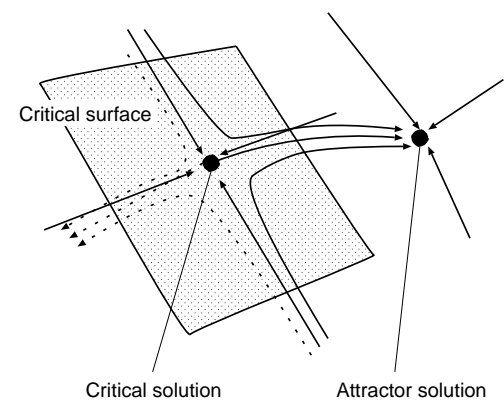

Figure 5. Schematic figure of qualitative properties of dynamical evolution of spacetimes with a self-similar attractor and a self-similar critical solution.

This example is also interesting in the context of dynamical properties of spacetimes in collapsing situations. This is actually the first demonstration of an attractor self-similar solution in generic spherical collapse in general relativity. The critical behaviour and selfsimilar attractor in spherical gravitational collapse suggest the validity of the so-called self-similarity hypothesis [24], i.e., the expectation that self-similar solutions can describe the asymptotic or intermediate behaviour of more general solutions in a variety of systems. 
For example, when we have both a self-similar attractor and a self-similar critical solution, which is the case for the spherical system of a perfect fluid with the equation of state $P=k \rho(0<k \leq 0.03)$, the qualitative properties of spacetime evolution can be depicted as in Fig. 5. The self-similarity hypothesis gives an important and useful view point for the general features of dynamical evolution of spacetimes through Einstein's field equations.

\subsection{Highly nonspherical collapse}

There are many other explicit examples of naked singularities, most of which are spherically symmetric. Although some of them provide an interesting point of view for gravitational collapse physics, unfortunately I cannot mention all of them because of space limitation. Here I would like to comment only on highly nonspherical collapse.

Among highly nonspherical collapse systems, the simplest one is cylindrically symmetric collapse. This system is described in time and one-dimensional space, which is the same as the spherically symmetric system. On the other hand, this system has one degree of freedom for gravitational waves, while the spherically symmetric system has none. The cylindrically symmetric system is the simplest system which contains both a collapsing body and gravitational waves. It has been proved that no horizon exists in this system $[25,26]$. This implies that if the cylindrical collapse ends in singularity, it must be naked. Cylindrically symmetric collapse models with and without (counter)rotation have been investigated by several authors [27-31]. A strong gravitational wave burst just before the singularity formation was reported [28]. I think many things are not fixed yet in the study of cylindrically symmetric collapse. I expect significant progress in the near future.

For axisymmetric case, we have a much less knowledge. The formation of singularity without apparent horizons has been reported numerically, which is called a spindle singularity $[32,33]$. However, it is well understood that a singularity without apparent horizon in some choice of time slicings does not necessarily mean a genuine naked singularity. It is still an open problem whether the spindle singularity reported by the result of numerical simulations is naked or not.

For completely general case, very little is known. Nevertheless we have a hoop conjecture [25]. The conjecture claims that black holes with horizons form when and only when a mass $M$ gets compacted into a region whose circumference in every direction is $\lesssim 4 \pi M$. It is interesting to note that this conjecture does not assume the cosmic censorship. There is no proof for or no counterexample against this conjecture. Recent progress in this conjecture has been made in higher dimensional gravity.

For highly nonspherical case, numerical relativity will give us a better insight in particular for cylindrically symmetric and axisymmetric cases. For completely general case, we will need extremely high performance computer because very high resolution will be necessary to solve the problem of spacetime singularities formed in gravitational collapse.

\section{Physics around naked singularities}

Since regions around naked singularities are regular, we can apply classical physics to these regions. Within the domain of dependence we can predict the evolution using classical physics. In principle we can expect something observable as a sign of extremely high 
curvature exposed to an observer. Here we see two examples, the evolution of nonspherical linear perturbation and the effect of quantum field theory in curved spacetime during the formation of naked singularities.

\subsection{Nonspherical perturbations and gravitational waves}

Most known examples of naked singularities formed in gravitational collapse are spherically symmetric. It is interesting to see whether these examples are stable or not against nonspherical perturbation. Nonspherical perturbations involve gravitational waves as seen in Fig. 6. Nonspherical metric and matter perturbations on spherically symmetric spacetimes are expanded by scalar, vector and tensor harmonics with quantum numbers $l$ and $m$. If we linearise these perturbations, the partial differential equations for gauge invariant perturbation quantities are obtained from Einstein's field equations and these perturbations are decomposed into two sectors with their parity.

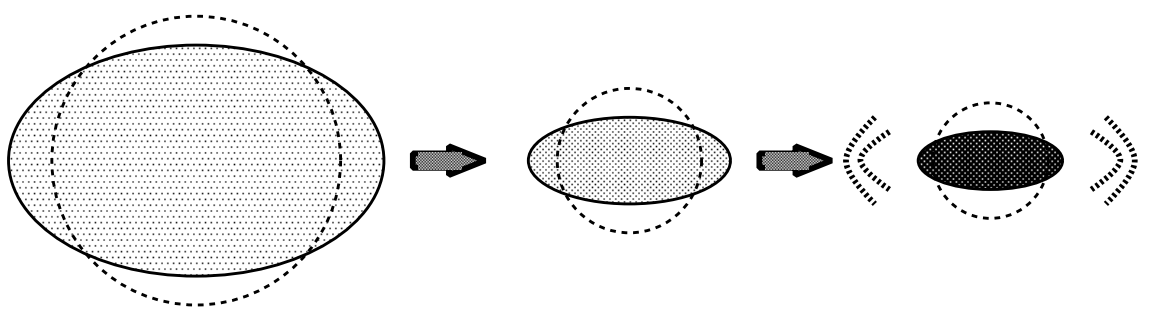

Figure 6. Schematic figure of nonspherical perturbations in spherical collapse background.

When we consider nonspherical perturbations of the LTB spacetime, the field equations for the perturbations result in a set of partial differential equations, among which main equations are wave equations in the LTB spacetime background. These equations are well integrated by numerical simulations up to near the Cauchy horizon. The result for $l=2$ is the following [34]. There is no significant growth of the energy flux of gravitational waves. On the other hand, some tetrad components of the Weyl curvature are diverging in an approach to the Cauchy horizon. The reason why this is possible is that the energy flux of gravitational waves comes from the first-order time derivative of the metric perturbation while the Weyl curvature is proportional to the second-order time derivative. The metric perturbations near the Cauchy horizon are described by the combination of the regular term and the term proportional to $\left(t_{C H}-t\right)^{\alpha}$, where $t_{C H}$ is the time of the Cauchy horizon appearance and $1<\alpha<2$. The diverging Weyl curvature will imply some instability of the Cauchy horizon.

The pure rotation of the body belongs to the odd-parity perturbation for $l=1$. In linear order analysis, we have no significant growth of perturbation on the Cauchy horizon. The centrifugal force due to rotation, which is essential for the collapse of a homogeneous dust ball, is the second-order effect. Even for $l=2$ perturbations, it is not clear how the diverging Weyl curvature in linear order analysis affects the Cauchy horizon. These considerations strongly suggest the importance of nonlinear analysis on nonspherical perturbations. 
This is one of the first systematic studies on nonspherical perturbations of spherically symmetric naked-singular spacetimes. It will be very interesting to study nonspherical perturbations of other examples of naked singularities or to adopt more general approach on the stability of naked singularity in spherically symmetric collapse against nonspherical perturbations. Important results have been obtained for the stability of critical solutions against nonspherical perturbations $[35,36]$.

\subsection{Quantum field theory in curved spacetime}

When quantum field theory is applied to gravitational collapse to a black hole, the constant thermal radiation, which is called the Hawking radiation, is derived. For naked-singular spacetimes, the extremely high-curvature region dynamically forms outside horizons. This fact suggests explosive particle creation around the forming naked singularity.

To estimate quantum particle creation, the Bogoliubov coefficients are calculated. For this purpose, we have to obtain mode functions in curved spacetimes. If we adopt the geometric optics approximation, this problem reduces to determining the 'double-null map' $v=G(u)$ between Eddington-Finkelstein outgoing and ingoing null coordinate $u$ and $v$ at the asymptotic region for outgoing and ingoing null rays, respectively, where the former is a reflection of the latter at the symmetric centre. This double-null map is obtained analytically for the self-similar LTB spacetime with the Schwarzschild exterior [37,38]. The resultant energy flux due to the particle emission is diverging in an approach to the Cauchy horizon. The spectrum of the emission is different from thermal radiation.

For two-dimensional case, the geometrical optics approximation is exact and an unambiguous expression is available for the quantum stress-energy tensor. The two-dimensional analogue of the four-dimensional LTB spacetime is obtained by removing the angular sector from the metric tensor. Then the physical mechanism of this particle emission can be discussed using the expectation value of the stress-energy tensor of quantum fields [39]. In this model, the positive energy gradually gathers around the centre during the collapse, leaving the negative-energy region around the positive-energy central region. Just before the singularity formation, the accumulated positive energy around the centre is emitted as an outgoing flux at a burst. Schematically, Fig. 7 shows the energy flow around the naked singularity.

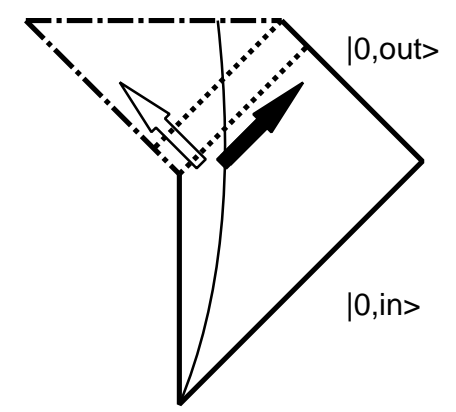

Figure 7. Schematic figure of quantum particle emission from a forming naked singularity. There are an outgoing positively diverging energy flux and an ingoing negatively diverging energy flux near the singularity. 
Although the self-similar LTB spacetime is simple and useful, it is somewhat unphysical because it does not admit Taylor expandable initial density profile at the centre. For the LTB spacetimes which admit Taylor expandable initial density profile, it is difficult to obtain the full analytic expression of the double-null map. Then, the double-null map was obtained numerically [40] and its qualitative property was also indicated analytically [41]. The resultant energy flux is also diverging in an approach to the Cauchy horizon.

The diverging energy flux along the Cauchy horizon suggests a possibility that the Cauchy horizon is unstable due to quantum effects and could be altered if the quantum gravity effects are seriously taken into account. However, there should be two major objections about these calculations. One objection is that the geometrical optics approximation has not been justified yet for the four dimensional LTB spacetimes. The difficulty come from the fact that quanta go through extremely curved region for naked singularity formation unlike for black hole formation. The other is that the amount of energy flux due to the particle creation is highly subjected to the cutoff scale if we introduce it. If we set the cutoff scale to be the Planck scale, the total radiated energy does not exceed the Planck energy [42]. Although we could extrapolate this perturbative calculation up to arbitrarily high energy scale, the calculated result has no justification if we do not have knowledge on physics beyond the cutoff scale. Because of these objections, I have to say that the divergent energy flux calculated in the LTB spacetimes is not sufficiently robust yet, even though it may be suggestive.

\subsection{Effective naked singularities}

Finally, I would like to propose the concept of 'effective naked singularities'. This has been coming up from the interaction of people in this field rather than is my own invention. For example, similar discussions have been made in Refs. [43,44]. See Ref [45] for the mathematical definition of this concept as the 'border of spacetime'.

For naked singularities, the curvature strength outside the horizons is typically diverging in an approach to the singularity. The cosmic censorship claims that naked singularities are not generic outcome of gravitational collapse of physically reasonable matter fields in classical general relativity.

However, it is now reasonable to consider that classical general relativity has a limitation towards the high energy end. We can consider a cutoff energy scale beyond which classical general relativity gives no longer good approximation and quantum gravity should be needed. Then it is natural to modify the classical notion of naked singularities. Let us consider that a spacetime region outside horizons. In this region, we can measure the energy scale of the spacetime by the curvature strength even if the spacetime is empty. If this energy scale is beyond the cutoff scale of classical general relativity, classical general relativity is not valid there and the spacetime should be modified considerably due to the quantum gravity effects. I will call this region an effective naked singularity. Figure 8 gives an illustration of an effective naked singularity.

As we have seen, we have already examples of naked singularities which form in the collapse of physically reasonable matter fields, although they may not be stable. The important implication of effective naked singularities is that they appear with nonzero probability in the collapse of physically reasonable matter fields. This is because we can realise the appearance of effective naked singularities if we prepare an initial data set which is suf- 


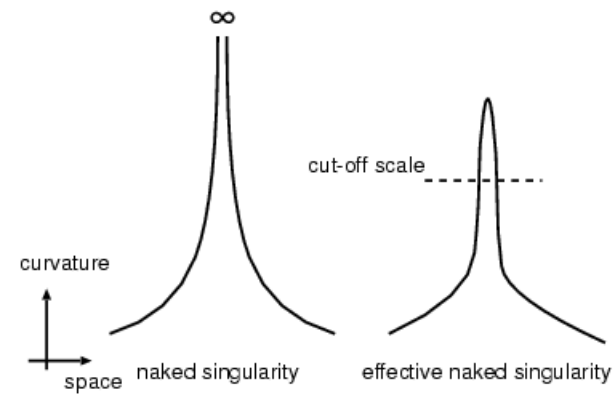

Figure 8. Schematic figure of effective naked singularities.

ficiently close to the one corresponding to naked singularity appearance. Since effective naked singularities are outside horizons, we can no longer predict our future without quantum gravity. In this sense, we can claim that the cosmic censorship conjecture is effectively violated, i.e., classical physics which does not include quantum gravity has a limitation in its future predictability with nonzero probability.

\section{Summary}

We have now a large number of examples of naked singularities. Among them, we can regard the black hole threshold and the self-similar attractor as naked singularities formed in the collapse of physically reasonable matter fields. The black hole threshold is unstable and the stability of the self-similar attractor against nonspherical perturbations is not clear. Therefore, it is still uncertain whether or not naked singularities are censored in physically reasonable gravitational collapse. However, these modern examples of naked singularities strongly suggest that effective naked singularities appear with nonzero (maybe not too small) probability for physically reasonable matter fields. Therefore, naked singularities are within the scope of physics. Naked singularities are worth studying in classical physics and in some form of quantum gravity, as the appearance of extremely strong curvature is in principle observable.

\section{References}

[1] S. W. Hawking and G. F. R. Ellis, The Large Scale Structure of Space-Time, (Cambridge University Press, Cambridge, England, 1973).

[2] C. J. S. Clarke, The Analysis of Space-Time Singularities, (Cambridge University Press, Cambridge, England, 1993).

[3] R. Penrose, Rivista del Nuovo Cimento, Numero Speziale I, 257 (1969); reprinted in Gen. Relat. Gravit. 34, 1141 (2002).

[4] R. Penrose, in General Relativity, an Einstein Centenary Survey, ed. S.W. Hawking and W. Israel, (Cambridge University Press, Cambridge, England, 1979), p.581.

[5] J. R. Oppenheimer and H. Snyder, Phys. Rev. 56, 455 (1939).

[6] G. Lemaître, Ann. Soc. Sci. Bruxelles A 53, 51 (1933); R. C. Tolman, Proc. Nat. Acad. Sci. 20, 169 (1934); H. Bondi, Mon. Not. R. astron. Soc. 107, 410 (1947). 
[7] D. M. Eardley and L. Smarr, Phys. Rev. D 192239 (1979).

[8] D. Christodoulou, Commun. Math. Phys. 93171 (1984).

[9] P. S. Joshi and I. H. Dwivedi, Phys. Rev. D 47, 5357 (1993); T. P. Singh and P. S. Joshi, Class. Quantum Grav. 13, 559 (1996); S. Jhingan and P. S. Joshi, Annals of Israel Physical Society Vol. 13, 357 (1997).

[10] R. P. A. C. Newman, Class. Quantum Grav. 3, 527 (1986).

[11] S. S. Deshingkar, P. S. Joshi and I. H. Dwivedi, Phys. Rev. D 59, 044018 (1999).

[12] F. C. Mena and B. C. Nolan, Class. Quantum Grav. 18, 4531 (2001).

[13] S. S. Deshingkar, P. S. Joshi and I. H. Dwivedi, Phys. Rev. D 65, 084009 (2002).

[14] M. W. Choptuik, Phys. Rev. Lett. 70, 9 (1993).

[15] A. M. Abrahams and C. R. Evans, Phys. Rev. Lett. 70, 2980 (1993).

[16] C. R. Evans and J. S. Coleman, Phys. Rev. Lett. 72, 1782 (1994).

[17] D. W. Neilsen and M. W. Choptuik, Class. Quantum Grav. 17, 761 (2000).

[18] T. Koike, T. Hara and S. Adachi, Phys. Rev. Lett. 74, 5170 (1995).

[19] J. M. Martín-García and C. Gundlach, Phys. Rev. D 68, 024011 (2003).

[20] T. Harada and H. Maeda, Phys. Rev. D 63, 084022 (2001).

[21] A. Ori and T. Piran, Phys. Rev. Lett. 59, 2137 (1987); Phys. Rev. D 42, 1068 (1990).

[22] T. Harada, Class. Quantum Grav. 18, 4549 (2001).

[23] T. Harada, Phys. Rev. D 58, 104015 (1998).

[24] B. J. Carr and A. A. Coley, Class. Quantum Grav. 16, R31 (1999).

[25] K. S. Thorne, in Magic Without Magic, ed. J.R. Klauder (Freeman, San Francisco, USA, 1972), p 231.

[26] S. A. Hayward, Class. Quantum Grav. 17, 1749 (2000).

[27] T. A. Apostolatos and K. S. Thorne, Phys. Rev. D 46, 2435 (1992).

[28] F. Echeverria, Phys. Rev. D 47, 2271 (1993).

[29] T. Chiba, Prog. Theor. Phys. 95, 321 (1996).

[30] P. R. C. T. Pereira and A. Wang, Phys. Rev. D 62, 124001 (2000).

[31] B. C. Nolan, Phys. Rev. D 65, 104006 (2002).

[32] T. Nakamura, K. Maeda, S. Miyama and M. Sasaki, in Proceedings of the 2nd Marcel Grossmann Meeting on General Relativity, ed. R. Ruffini (North-Holand, Amsterdam, Netherlands, 1982), p. 675 .

[33] S. L. Shapiro and S. A. Teukolsky, Phys. Rev. Lett. 66, 994 (1991); Phys. Rev. D 45, 2006 (1992).

[34] H. Iguchi, K. Nakao and T. Harada, Phys. Rev. D 57, 7262 (1998); H. Iguchi, T. Harada and K. Nakao, Prog. Theor. Phys. 101, 1235 (1999); Prog. Theor. Phys. 103, 53 (2000).

[35] J. M. Martín-García and C. Gundlach, Phys. Rev. D 59, 064031 (1999).

[36] C. Gundlach, Phys. Rev. D 65, 084021 (2002).

[37] S. Barve, T. P. Singh, C. Vaz and L. Witten, Nucl. Phys. B 532, 361 (1998); Phys. Rev. D 58, 104018 (1998).

[38] C. Vaz and L. Witten, Phys. Lett. B 442, 90 (1998).

[39] H. Iguchi and T. Harada, Class. Quantum Grav. 18, 3681 (2001).

[40] T. Harada, H. Iguchi and K. Nakao, Phys. Rev. D 61, 101502(R) (2000); Phys. Rev. D 62, 084037 (2000).

[41] T. Tanaka and T. P. Singh, Phys. Rev. D 63, 124021 (2001).

[42] T. Harada, H. Iguchi, K. Nakao, T. P. Singh, T. Tanaka and C. Vaz, Phys. Rev. D 64, 041501(R) (2001).

[43] P. S. Joshi, Global aspects in gravitation and cosmology, (Oxford University Press, Oxford, England, 1993).

[44] S. Jhingan, $\mathrm{PhD}$ thesis (1998).

[45] T. Harada and K. Nakao, Phys. Rev. D 70, 041501(R) (2004). 